\title{
PERSISTENCIA DEL ENDOSULFÁN EN MEDIO ACUOSO ESTÁTICO
}

\author{
ARGELIA M. L. LENARDÓN* \\ PATRICIA M. DE LA SIERRA * \\ FERNANDA MARINO ***
}

\begin{abstract}
Estudou-se a cinética de degradação da mistura dos isômeros alfa e beta Endosulfan em diferentes condições de trabalho. Os compostos foram adicionados em água ultrapura, água do rio, água de rio filtrada e água ultrapura com sais (salinidade similar à agua do rio utilizada). As condições de degradação escolhidas foram: escuridão e duas temperaturas $\left(14 \pm 1^{\circ} \mathrm{C}\right.$ e $\left.26 \pm 1^{\circ} \mathrm{O}\right)$. As amostragens foram programadas de modo a se obter dados periódicos mais freqüentes no início da experiência e posteriormente mais espaçados até o seu final (230 dias). As amostras foram submetidas à microextração e analisadas por cromatografia em fase gasosa com detector de $\mathrm{Ni}^{63}$ e coluna Megabore DB-5. A degradação foi descrita de acordo com a cinética de primeira ordem, determinando-se os tempos de meia vida ( $\mathrm{t} 1 / 2)$ e as energias de ativação $(\mathrm{Ea})$. Os dados obtidos evidenciaram que a temperatura é o fator preponderante, sendo possível deduzir que o alfaendosulfan, exceto para água ultrapura $(A U)$, é mais influenciado pela temperatura do que o beta-endosulfan. $O$ segundo efeito mais importante refere-se ao tipo de água utilizada como matriz, devido à influência da salinidade.
\end{abstract}

PALAVRAS-CHAVE: PESTICIDAS - DEGRADAÇÃO; CROMATOGRAFIA A GÁS; ENDOSULFAN.

* Dra. en Química, Investigador Científico del Consejo Nacional de Investigaciones Científicas y Técnicas (CONICET), Prof. De la Universidad Nacional del Litoral (UNL), Santa Fe, Argentina.

** Lic. en Química, Profesional Adjunto del CONICET, Prof. de la UNL, Santa Fe, Argentina.

*** Téc. Química, Técnico Principal del CONICET, Santa Fe, Argentina. (e-mail: fmarino@ceride.gov.ar). 


\section{INTRODUCCIÓN}

El Endosulfán (6,7,8,9,10,10-hexacloro-1,5,5a,6,9,9a-hexahydro6,9-metano-2,4,3-benza-dioxatiepin 3-oxide) es un insecticida y acaricida organoclorado del subgrupo del ciclodieno utilizado para el control de plagas en una amplia variedad de alimentos. Actua por contacto, ingestión y vaporización, acelerados por temperaturas elevadas y alta humedad relativa. Se utiliza en cultivos tales como: algodón, papa, pimiento, tomate y tabaco (1).

Se estudia a los isómeros alfa y beta Endosulfán ya que son los que están presentes en los formulados técnicos (alfa-Endosulfán se encuentra en mayor proporción que beta-Endosulfán).

Ambos isómeros son altamente tóxicos para la vida acuática y la salud humana por lo que es importante conocer su cinética de degradación bajo distintas condiciones del medio. En la Tabla 1, se muestran algunas propiedades de los isómeros alfa y beta.

\section{TABLA 1 - PROPIEDADES FÍSICAS Y TOXICOLÓGICAS}

\begin{tabular}{lcc}
\hline Propiedades & alfa-Endosulf/戶্ & beta-Endosulf/厚 \\
\hline Solubilidad en agua $(\mathrm{mg} / \mathrm{L})$ a 22"C & 0.32 & 0.33 \\
Punto de fusi $\mathrm{n}($ "C) & 109 & 213 \\
$\mathrm{LD}_{50}$ (ratas $\left.\mathrm{mg} / \mathrm{kg}\right)$ & 76 & 240 \\
\hline
\end{tabular}

La persitencia de los pesticidas en agua puede ser evaluada por tres procesos de degradación: microbiológico, químico y fotoquímico. Muchos autores examinan la cinética de degradación de una amplia gama de biocidas bajo distintas ópticas (2-6). En este trabajo se estudió la cinética de degradación química de una mezcla de alfa y beta-Endosulfán en distintos tipos de agua y a distintas temperaturas. Para evaluar los tiempos de vida media ( $\left.\mathrm{t}_{1 / 2}\right)$ y las energías de activación $\left(\mathrm{E}_{\mathrm{a}}\right)$ se trabajó con distintos tipos de agua: agua ultrapura $(A U)$, agua de río (AR), agua de río filtrada (ARF) y agua ultrapura adicionada con sales (AA) para imitar una salinidad similar al agua de río, utilizando el método descripto por GUNNISON et al. (7); y a dos temperaturas: $14 \pm 1^{\circ} \mathrm{C}$ y $26 \pm 1^{\circ} \mathrm{C}$. Los ensayos con diferentes tipos de agua se realizaron con el objetivo de 
estudiar las diferencias en el proceso de degradación que son consecuencia de la presencia de sustancias disueltas (orgánicas y/ó inorgánicas) y material particulado.

Si bien es difícil predecir la persistencia de los plaguicidas en el medio ambiente debido a las dificultades de extrapolar los resultados de degradación obtenidos bajo condiciones controladas, estas nos proporcionan información indispensable para aproximarnos a la situación real.

\section{MATERIALES Y MÉTODOS}

\subsection{MATERIALES}

Los estándares de pesticidas utilizados: alfa-Endosulfán y betaEndosulfán provienen del Lab. Dr. Ehrenstorfer (proveedor EUROBASE S.p.A. Milano, Italia) y su pureza es: $99.2 \%$. Se utilizó agua ultrapura calidad HPLC (Fisher Scientific), hexano calidad plaguicida (Sintorgan) y reactivos químicos (Merck) calidad proanálisis.

\subsection{TIPOS DE AGUA}

\section{- $\quad$ Agua ultrapura (AU)}

Se probaron cromatográficamente agua destilada, agua destilada y desionizada y agua calidad HPLC (Fisher Scientific). Se utilizó ésta última como matríz para las experiencias, ya que no presenta interferencias cromatográficas.

\section{- Agua de río (AR)}

Se tomó una muestra en el río Paraná a la altura de la ciudad de Santa Fe (Argentina). Esta zona se encuentra ubicada en el centro de la pampa húmeda donde la agricultura y la ganadería son intensas, por lo que tanto el río como sus afluentes son objeto de constantes monitoreos. Esta muestra se analizó por cromatografía gaseosa para descartar la presencia de alfa y beta-Endosulfán.

\section{- Agua de río fitrada (ARF)}

Al agua de río se la filtró con filtros Wathman GF/C. La cantidad de sólidos suspendidos es $25 \mathrm{mg} / \mathrm{L}$. 


\section{- Agua adicionada con sales (AA)}

Se adicionaron sales al agua ultrapura logrando una concentración promedio de iones similar al agua de río proveniente de la zona de muestreo. Las sales adicionadas fueron: cabonato ácido de sodio, sulfato de magnesio heptahidratado, cloruro de potasio, fosfato diácido de potasio, nitrato de potasio, cloruro férrico hexahidratado y cloruro de calcio.

Todos los tipos de agua se esterilizaron a $120^{\circ} \mathrm{C}$ en autoclave durante 20 minutos y luego se burbujearon con nitrógeno durante 10 minutos. En la Tabla 2, se observan las características de cada tipo de agua.

\section{TABLA 2 - CARACTERÍSTICAS DE CADA TIPO DE AGUA}

\begin{tabular}{lcccc}
\hline PAR METRO & AU & AR & ARF & AA \\
\hline PH & 6,90 & 7,00 & 6,90 & 7,10 \\
Conductividad $\left(\mu \mathrm{S} / \mathrm{cm} 25^{\circ} \mathrm{C}\right)$ & $<0,06$ & 151 & 148 & 134 \\
Residuos a $105^{\circ} \mathrm{C}(\mathrm{mg} / \mathrm{L})$ & $<1$ & 98 & 73 & 72 \\
Cloruros $\left(\mathrm{mg} \mathrm{L}^{-1}\right)$ & $<0,1$ & 25 & 23 & 23 \\
Sulfatos $\left(\mathrm{mg} \mathrm{L}^{-1}\right)$ & $<0,1$ & 33 & 32 & 18 \\
Nitratos $\left(\mathrm{mg} \mathrm{L}^{-1}\right)$ & $<1,0$ & $<1,0$ & $<1,0$ & $<1,0$ \\
Nitritos $\left(\mathrm{mg} \mathrm{L}^{-1}\right)$ & $<0,01$ & $<0,01$ & $<0,01$ & $<0,01$ \\
Calcio $\left(\mathrm{mg} \mathrm{L}^{-1}\right)$ & $<1,0$ & 8 & 8 & 7 \\
Magnesio $\left(\mathrm{mg} \mathrm{L}^{-1}\right)$ & $<0,02$ & 4 & 3 & 4 \\
Sodio $\left(\mathrm{mg} \mathrm{L}^{-1}\right)$ & $<0,03$ & 15 & 14 & 13 \\
Potasio $\left(\mathrm{mg} \mathrm{L}^{-1}\right)$ & $<0,1$ & 4 & 4 & 3 \\
Dureza total $\left(\mathrm{mg} \mathrm{L}^{-1} \mathrm{CaCO}_{3}\right)$ & $<1,0$ & 35 & 32 & 27 \\
\hline
\end{tabular}

$\mathrm{AU}=$ Agua ultrapura; $\mathrm{AR}=$ Agua de río; $\mathrm{ARF}$ = Agua de río filtrada; $\mathrm{AA}=$ Agua adicionada con sales.

\subsection{CONDICIONES DE DEGRADACIÓN}

Se prepararon soluciones stock de cada isómero en acetona de concentración aproximada: $20 \mu \mathrm{g} / \mu \mathrm{L}$. Se colocaron $20 \mu \mathrm{L}$ de cada una de las soluciones stock en un matráz de $5 \mathrm{~mL}$ y se llevó a volumen con 
hexano (solución de siembra). Las concentraciones en ésta mezcla resultaron aproximadamente $0,08 \mu \mathrm{g} / \mu \mathrm{L}$.

De ésta solución se sembraron $200 \mu \mathrm{L}$ a cada frasco. Luego de evaporar el solvente en los frascos bajo corriente de nitrógeno, se les agregó a cada uno un volumen de $250 \mathrm{~mL}$ de la muestra ó blanco correspondiente, con lo que las concentraciones iniciales para la experiencia resultaron: alfa-Endosulfán: $62,3 \mathrm{pg} / \mu \mathrm{L}$ y beta-Endosulfán: $64,0 \mathrm{pg} / \mu \mathrm{L}$. Las muestras sembradas se sometieron a las condiciones de degradación previstas: 14 y $26^{\circ} \mathrm{C}$ en oscuridad, utilizando incubadores con regulación de $\pm 1^{\circ} \mathrm{C}$.

Los frascos se dejaron 24 horas en reposo y luego se comenzó el análisis de los residuos tomando alícuotas en el tiempo cero, 4, 6 y 10 días, para continuar después cada 10 días hasta dar por finalizada la experiencia. Se dio por concluído el trabajo a los 230 días, cuando en todos los casos quedaba un remanente menor al $50 \%$ de la concentración inicial para poder evaluar convenientemente los tiempos de vida media.

\subsection{EXTRACCIÓN}

Se evaluaron métodos que proponen el uso de bajas cantidades de solvente, como por ejemplo el propuesto por ONG et al. (8). Se optó por una microextracción líquido-líquido. Para tal fin se hizo una selección de solventes de distinta polaridad (hexano, benceno, tolueno, acetona), de la relación solvente/muestra y de los tiempos de agitación apropiados (5, 10 y 30 minutos). El método elejido finalmente consiste en extraer $200 \mu \mathrm{L}$ de muestra con $1 \mathrm{~mL}$ de hexano en viales de vidrio de $2 \mathrm{~mL}$ de capacidad con septa en la tapa, agitar durante 5 minutos, dejar la muestra en reposo 20 minutos y finalmente cromatografiar $1 \mu \mathrm{L}$ de la capa orgánica.

\subsection{ANÁLISIS CUANTITATIVOS}

Los pesticidas se identificaron y cuantificaron por cromatografía gaseosa. El equipo utilizado fue un cromatógrafo VARIAN 3400 con inyector automático, detector de captura electrónica y columna Megabore DB-5 (30 m x $0.53 \mathrm{~mm}$ I.D., $1.5 \mu \mathrm{m}$ ). Las condiciones operativas fueron: temperatura de inyector $240{ }^{\circ} \mathrm{C}$, temperatura de detector $320^{\circ} \mathrm{C}$ y temperatura de columna $220^{\circ} \mathrm{C}$. Se utilizó el modo de inyección automático splitless (relación de split 10X) con un volumen de inyección de $1 \mu \mathrm{L}$. 


\subsection{ANÁLISIS DE RECUPERACIÓN}

Se analizaron las muestras sembradas por triplicado con el fin de comprobar la eficiencia del método de trabajo. Para ambos biocidas se obtienen buenos porcentajes de recuperación: entre un 87 y $98 \%$, con una desviación estándar relativa de 6-9\% (9).

\section{RESULTADOS Y DISCUSIÓN}

Para determinar la cinética de degradación de los biocidas en estudio, se graficó concentración versus tiempo y se realizó el analisis de regresión exponencial de cada grupo de datos. Se determinó la constante de velocidad $\mathrm{k}$ (Tabla 3 ) a través de la ecuación que describe una cinética de primer orden:

$$
C_{t}=C_{0} \cdot e^{-k t}
$$

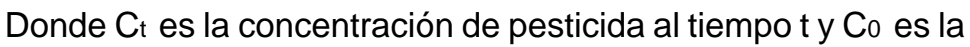
concentración inicial, ambas concentraciones expresadas en $\mathrm{pg} / \mu \mathrm{L}$; y $\mathrm{k}$ en días ${ }^{-1}$.

El tiempo de vida media, $\mathrm{t} 1 / 2$ (días), se determina para cada tipo de agua a las diferentes condiciones de degradación a partir de la ecuación de primer orden, reemplazando $\mathrm{Ct}$ por $\mathrm{Co} / 2$ resultando (Tabla 4).

$$
t_{1 / 2}=\ln 2 / k
$$

La velocidad de degradación es afectada por la temperatura y la dependencia de $\mathrm{k}$ con la temperatura esta dada por la ecuación de Arrhenius:

$$
k=A \cdot e^{-E a / R T}
$$

Donde $\mathrm{A}$ es el factor de frecuencia, $\mathrm{T}$ es la temperatura, $\mathrm{R}$ la constante de los gases y Ea la energía de activación.

Se realizaron gráficas de decaimiento de la concentración del compuesto expresada como porcentaje de la concentración inicial en función del tiempo transcurrido en días. Se analizó la importancia de dos efectos: tipo de agua y temperatura $\left(14{ }^{\circ} \mathrm{C}\right.$ y $\left.26^{\circ} \mathrm{C}\right)$.

Evaluando el tipo de agua se observan diferencias entre $\mathrm{AU}$ y AA, atribuibles a la salinidad del medio. En la Figura 1 vemos como la salinidad a mayor temperatura $\left(26^{\circ} \mathrm{C}\right)$ aumenta la cinética de degradación 
para ambos isómeros. En el caso del AR y ARF no existen diferencias significativas como se observa en la Figura 2.

\section{TABLA 3 - CONSTANTES DE VELOCIDAD DE REACCIÓN (k)}

\begin{tabular}{|c|c|c|c|}
\hline \multicolumn{2}{|c|}{ MUESTRA } & \multirow{2}{*}{ 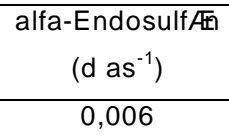 } & \multirow{2}{*}{ 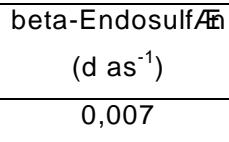 } \\
\hline $\mathrm{AU}$ & $14^{\circ} \mathrm{C}$ & & \\
\hline & $26^{\circ} \mathrm{C}$ & 0,009 & 0,013 \\
\hline \multirow[t]{2}{*}{$A R$} & $14^{\circ} \mathrm{C}$ & 0,014 & 0,021 \\
\hline & $26^{\circ} \mathrm{C}$ & 0,131 & 0,096 \\
\hline \multirow[t]{2}{*}{ ARF } & $14^{\circ} \mathrm{C}$ & 0,017 & 0,024 \\
\hline & $26^{\circ} \mathrm{C}$ & 0,084 & 0,139 \\
\hline \multirow[t]{2}{*}{ AA } & $14^{\circ} \mathrm{C}$ & 0,026 & 0,046 \\
\hline & $26^{\circ} \mathrm{C}$ & 0,277 & 0,111 \\
\hline
\end{tabular}

Los coeficientes de correlación varían entre 0.80 - 0.99 .

$\mathrm{AU}=$ Agua ultrapura; $\mathrm{AR}=$ Agua de río; $\mathrm{ARF}$ = Agua de río filtrada; $\mathrm{AA}=$ Agua adicionada con sales.

\section{TABLA 4 - TIEMPOS DE VIDA MEDIA ( $\left.\mathbf{t}_{1 / 2}\right)$}

\begin{tabular}{|c|c|c|c|c|c|}
\hline & $\begin{array}{c}\text { Temperatura } \\
\left({ }^{\circ} \mathrm{C}\right)\end{array}$ & $\begin{array}{c}\mathrm{AU} \\
\text { (d as) }\end{array}$ & $\begin{array}{c}\text { AR } \\
\text { (d as) }\end{array}$ & $\begin{array}{l}\text { ARF } \\
\text { (d as) }\end{array}$ & $\begin{array}{c}\text { AA } \\
\text { (d as) }\end{array}$ \\
\hline \multirow[t]{2}{*}{ alfa-Endosulf/压 } & 14 & 116 & 50 & 41 & 27 \\
\hline & 26 & 77 & 5 & 8 & 3 \\
\hline \multirow[t]{2}{*}{ 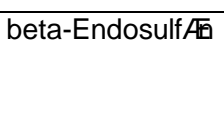 } & 14 & 99 & 33 & 29 & 15 \\
\hline & 26 & 53 & 7 & 5 & 6 \\
\hline
\end{tabular}

$\mathrm{AU}=$ Agua ultrapura; $\mathrm{AR}=$ Agua de río $; \mathrm{ARF}=$ Agua de río filtrada; $\mathrm{AA}=$ Agua adicionada con sales. 


\section{FIGURA 1 - INFLUENCIA DE LA SALINIDAD EN EL DECAIMIENTO DEL ENDOSULFÁN}
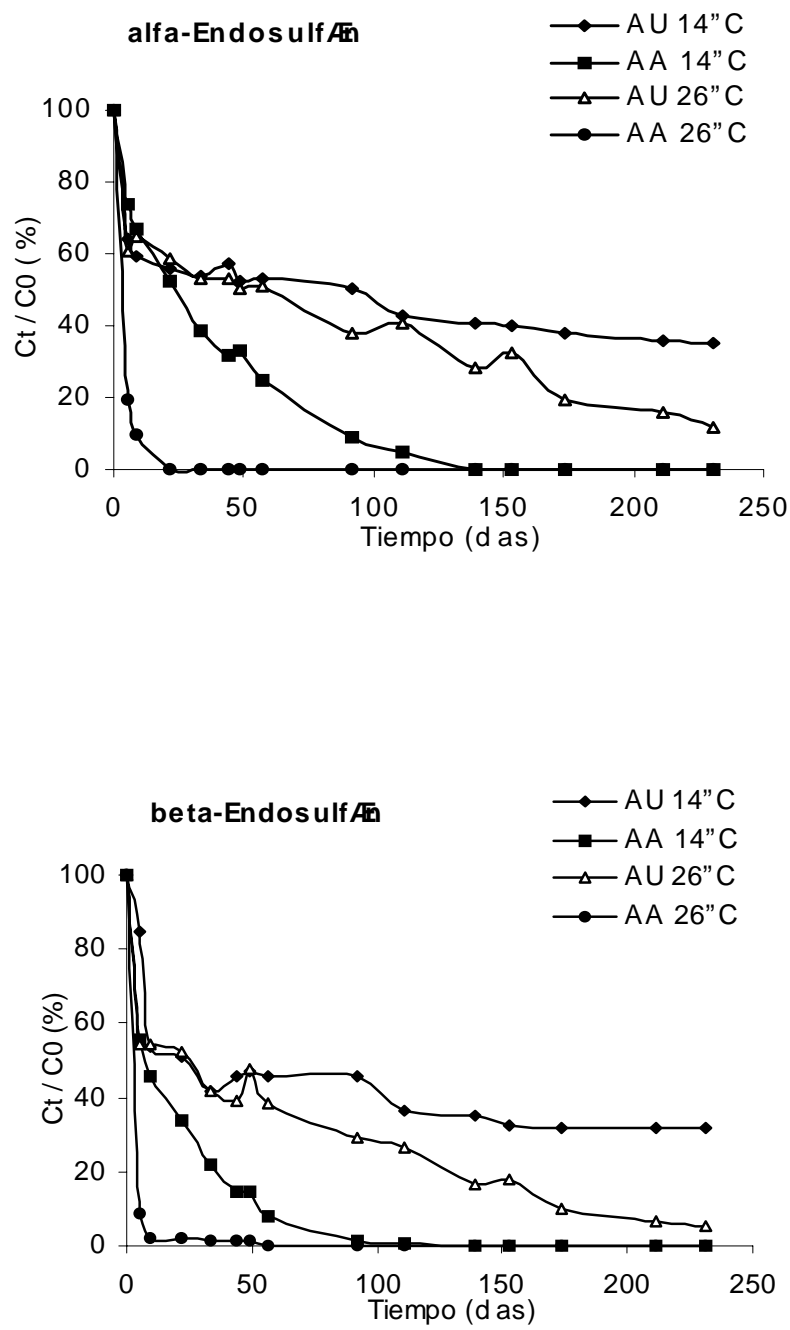

$\mathrm{Ct}=$ concentración residual al tiempo $\mathrm{t}$.

$\mathrm{C} 0=$ concentración inicial. 


\section{FIGURA 2 - DECAIMIENTO DEL ENDOSULFÁN EN AGUA DE RÍO ENTERA Y FILTRADA}

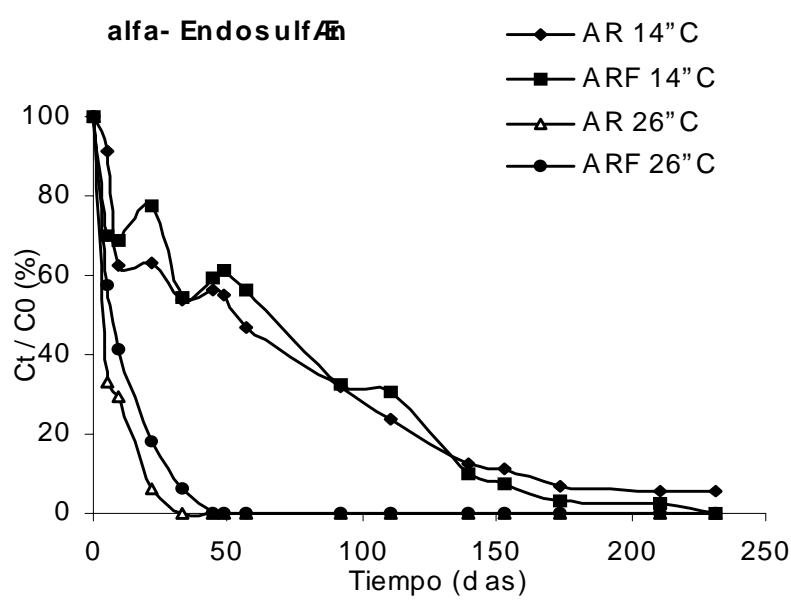

be ta-Endos u If 质

A R $14 " \mathrm{C}$

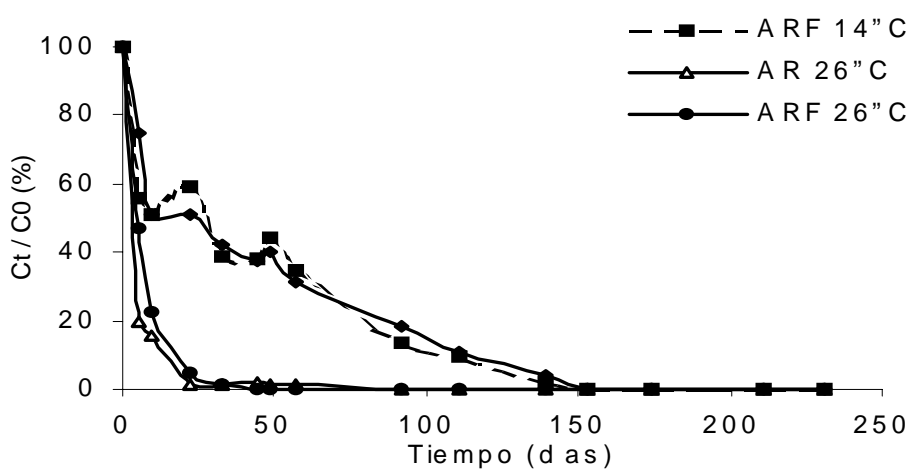

$\mathrm{Ct}=$ concentración residual al tiempo $\mathrm{t}$.

$\mathrm{CO}=$ concentración inicial. 
Los tiempos de vida media a $14{ }^{\circ} \mathrm{C}$ presentan valores levemente más bajos para el beta-Endosulfán que para el alfa-Endosulfán y a $26 \stackrel{\circ}{ } \mathrm{C}$ las diferencias no son notables Figura 3.

\section{FIGURA 3 - TIEMPOS DE VIDA MEDIA PARA LOS ISÓMEROS ALFA Y BETA ENDOSULFÁN PARA TODAS LAS VARIABLES ANALIZADAS}

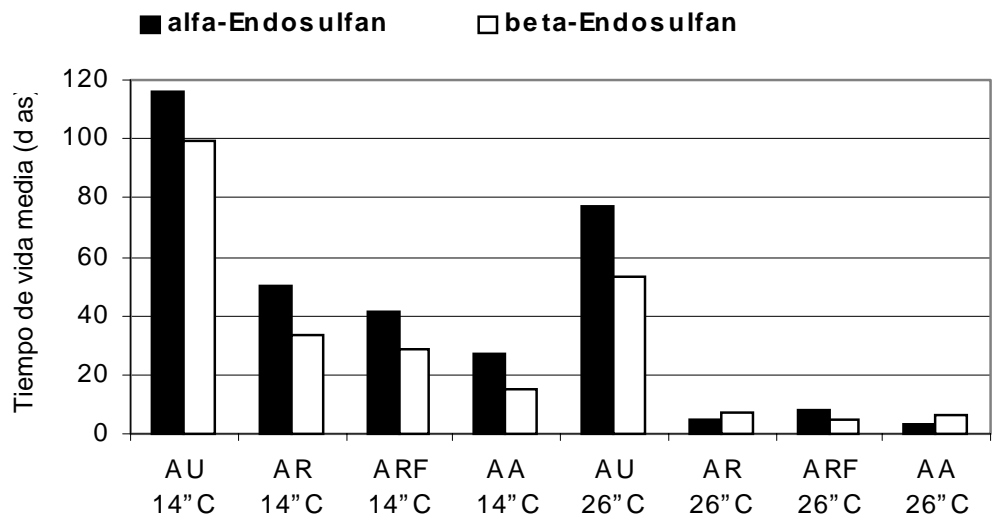

$\mathrm{AU}=$ Agua ultrapura; $\mathrm{AR}=$ Agua de río; $\mathrm{ARF}=$ Agua de río filtrada; $\mathrm{AA}=$ Agua adicionada con sales.

Los valores de Ea demuestran la influencia de la temperatura con la cinética de degradación; a mayores valores de Ea mayor dependencia con la temperatura. El aumento de la temperatura acelera la cinética de degradación de los isómeros, pero dicho comportamiento depende del tipo de agua (Tabla 5).

TABLA 5 - ENERGÍAS DE ACTIVACIÓN (Ea)

\begin{tabular}{cccc}
\hline $\mathrm{AU}$ & $\mathrm{AR}$ & $\mathrm{ARF}$ & $\mathrm{AA}$ \\
$(\mathrm{kcal} / \mathrm{mol})$ & $(\mathrm{kcal} / \mathrm{mol})$ & $(\mathrm{kcal} / \mathrm{mol})$ & $(\mathrm{kcal} / \mathrm{mol})$
\end{tabular}

\begin{tabular}{|c|c|c|c|c|}
\hline alfa-Endosulff任 & 0.2 & 31.8 & 22.7 & 33.6 \\
\hline beta-Endosulf/俰 & 8.8 & 21.6 & 25.0 & 12.5 \\
\hline
\end{tabular}

$\mathrm{AU}=$ Agua ultrapura; $\mathrm{AR}=$ Agua de río $; \mathrm{ARF}=$ Agua de río filtrada; $\mathrm{AA}=$ Agua adicionada con sales. 
Analizando las energías de activación para los distintos tipos de agua (exceptuando $\mathrm{AU}$ ) podemos afirmar que no existen diferencias entre los dos isómeros. Las bajas energías de activación que presentan tanto el alfa como el beta-Endosulfán en AU confirman lo dicho anteriormente: la cinética de degradación es influenciada por el tipo de agua y por la temperatura.

Ambos isómero presentan propiedades físicas similares (Tabla 1), esto se corresponde con la similar persistencia a $26^{\circ} \mathrm{C}$, sin embargo a $14{ }^{\circ} \mathrm{C}$ el alfa-Endosulfan presenta mayor tiempos de vida media que el beta-Endosulfán en los cuatro tipos de agua.

\section{CONCLUSIÓN}

Los resultados demuestran que los plaguicidas en estudio siguen una cinética de primer orden. Evaluando el factor temperatura observamos que el beta-Endosulfán presenta tiempos de vida media inferiores al isómero alfa, esto es resultado de su mayor degradación en un sistema controlado. Las diferencias en la degradación de ambos isómeros no son significativas.

Analizando el tipo de agua, la salinidad del medio juega un papel importante, esto se podría atribuir a un disminución de la solubilidad de los plaguicidas favoreciendo la velocidad de degradación, lo que se demuestra con los bajos tiempos de vida media del agua adicionada con sales comparados con los correspondientes a otros tipos de matrices.

\section{Abstract}

\section{PERSISTANCE OF ENDOSULFAN IN STATIC AQUEOUS MEDIUM}

Degradation kinetics of a mixture of alpha- and beta-Endosulfan isomers was studied under different conditions. The compounds were spiked in ultrapure water, river water, filtered water and ultrapure water with salts (similar salinity condition to that of the river water used). The degradation conditions chosen were: darkness, two temperatures $\left(14 \pm 1^{\circ} \mathrm{C}\right.$ e $\left.26 \pm 1^{\circ} \mathrm{C}\right)$. Samplings were programmed in order to obtain more frequent periodical data in the beginning of the experience and after more spaced until its end (230 days). The samples were submitted to microextraction and then analyzed by gas chromatography through a $\mathrm{Ni}^{63}$ detector equipped with a Megabore DB-5 column. Degradation was described using first-order kinetics to determine half-life times ( $t 1 / 2)$ and activation energies $\left(E_{a}\right)$. The data obtained evidenced that temperature is the predominant factor, it can possibly be inferred that alfa-endosulfan is much more influenced than beta-endosulfan except for ultrapure water (UW). The second important effect is the water type used as matrix, due to the influence of salinity. 
KEY-WORDS: PESTICIDES - DEGRADATION; GAS CHROMATOGRAPHY; REFERÊNCIAS ENDOSULFAN.

1 CÁMARA DE SANIDAD AGROPECUARIA Y FERTILIZANTES DE LA REPÚBLICA ARGENTINA. Guía de productos fitosanitarios para la República Argentina. Buenos Aires (Argentina): CASAFE, 1997. $1600 \mathrm{p}$.

2 LARTIGUES, S.B.; GARRIGUES, P.P. Degradation kinetics of organophosphorus and organonitrogen pesticides in different waters under various environmental conditions. Environ. Sci. \& Technol., v. 29, n. 5, p. 1246-54, 1995.

3 WANG, T.C.; HOFFMAN, M.E. Degradation of organophosphorus pesticides in coastal water. J. Assoc. Off. Anal. Chem., v. 74, n. 5, p. 883-86, 1991.

4 SAENZ-ASENSIO, J., PLAZA-MEDINA, M.; MARTÍNEZ-SORIA, M.T. Kinetic study of the degradation of ethiofencarb in aqueous solutions. Pestic. Sci., v. 50, Issue 3, p. 187-94, 1997.

5 FREED, V.H., CHIOU, C.T.; SCHMEDDING, D.W. Degradation of selected organophosphate pesticides in water and soil. J. Agric. Food Chem., v. 27, n. 4, p. 706-08, 1979.

6 DURAND, G.; BARCELÓ, D.; ALBAIGÉS, J.; MANSOUR, M. Utilisation of liquid cromatography in aquatic photodegradation studies of pesticides: a comparison between distilled water and seawater. Cromatographia, v. 29, n. 3/4, p. 120-124, 1990.

7 GUNNISON, D.; BRANNON, J.M.; CHEN, R.L., SMITH, I.; STURGIS, T.C. Environmental and water quality operational studies. USA: US Army Corps of Engineers, 1984. 60 p. (E-84-2). (Miscellaneous Paper).

8 ONG, V.S.; HITES, R.A. Determination of pesticides and polychlorinated biphenyls in water: a low-solvent method. Environ. Sci. Technol., v. 29, n. 5, p. 1259-1266, 1995.

9 MILLER, J.C.; MILLER, J.C. Estadística para química analítica. 2. ed. USA: Ed. Addison-Wesley Iberoamericana, 1993. p. 211. 